\title{
Balkanologie
}

Balkanologie Revue d'études pluridisciplinaires

Vol. $15 n^{\circ} 1 \mid 2020$

Mémoires performatives : faire des passés et des présents

\section{David Montgomery, Everyday Life in the Balkans}

Bloomington, Indiana University Press, 2019

\section{Mehdi Belasri}

\section{(2) OpenEdition}

\section{Journals}

Édition électronique

URL : https://journals.openedition.org/balkanologie/2543

DOI : 10.4000/balkanologie.2543

ISSN : 1965-0582

Éditeur

Association française d'études sur les Balkans (Afebalk)

Référence électronique

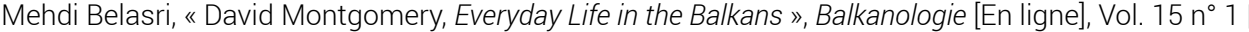
2020, mis en ligne le 01 juin 2020, consulté le 05 août 2021. URL : http://journals.openedition.org/ balkanologie/2543; DOI : https://doi.org/10.4000/balkanologie.2543

Ce document a été généré automatiquement le 5 août 2021.

(C) Tous droits réservés 


\section{David Montgomery, Everyday Life in the Balkans}

Bloomington, Indiana University Press, 2019

Mehdi Belasri

\section{RÉFÉRENCE}

Montgomery, David, 2019, Everyday Life in the Balkans, Bloomington, Indiana University Press, 422 pages, ISBN 9780253038173

1 David Montgomery, anthropologue de l'Université du Maryland, spécialiste du lien entre islam et vie quotidienne en Asie centrale et auteur de Practising Islam (2016) ${ }^{1}$ et Everyday Life in Central Asia (2007)2, nous invite à découvrir la vie quotidienne dans les Balkans à travers cet ouvrage qu'il a dirigé. Everyday Life in the Balkans rassemble les contributions de 35 chercheurs issus de différents champs des sciences sociales comme la sociologie, l'histoire ou l'anthropologie. La plupart des contributions se basent sur des enquêtes de terrain effectuées entre le début des années 2000 et la fin des années 2010 sur des terrains en Slovénie, Croatie, Serbie, Kosovo, Grèce, Bulgarie, Monténégro, Bosnie-Herzégovine, Roumanie, Albanie et Macédoine, avec un intérêt pour les cadres urbains et ruraux. L'ouvrage, de plus de 400 pages, s'organise autour de six thématiques ayant trait à la vie quotidienne dans les Balkans : le contexte historique de la vie quotidienne, les relations familiales et amicales, les moyens de subsistance, la place du politique dans la vie quotidienne, les religions abordées par leurs pratiques, les pratiques artistiques et les arts de faire au quotidien. L'ouvrage explore tour à tour le point de vue territorial, historique, culturel, cultuel ou économique pour aborder ces thématiques.

Ce n'est pas le premier ouvrage centré sur la vie quotidienne dans les Balkans. Parmi les travaux ayant exploré cette problématique, nous pouvons mentionner notamment les recherches de Piotr Goldstein sur la fabrique du politique au quotidien à Mostar et à Novi Sad ${ }^{3}$, celui de Breda Luthar et Maruša Pušnik sur la vie quotidienne en Yougoslavie 
socialiste ${ }^{4}$, les travaux de Johann Strauss sur la vie quotidienne à l'époque ottomane ${ }^{5}$ ou encore la recherche de Rory Archer sur les controverses autour de la Turbo Folk ${ }^{6}$. À la différence de ses prédécesseurs, Everyday Life in the Balkans est remarquable en raison du nombre et de la diversité des contributions rassemblées.

3 L'un des premiers objectifs de cet ouvrage collectif est de donner plus de profondeur au terme «Balkans ». Le terme est intentionnellement choisi car il est, comme l'explique David Montgomery, porteur d'interprétations et d'appréciations personnelles de la part $\mathrm{du}$ lecteur. Ainsi, ce terme renverrait directement aux représentations que l'on se fait des sociétés dans la région, contrairement à " Europe du Sud-Est », un terme neutre et n'apportant qu'un bornage géographique. L'ouvrage interroge les Balkans et leurs limites d'un point de vue historique à travers la contribution de l'historien Andrew Wachtol. L'auteur passe en revue ce que les recherches archéologiques et historiques peuvent nous dire de la vie quotidienne dans la région de la conquête romaine jusqu'à la période ottomane. D'autres contributions adoptent un point de vue contemporain sur l'identité balkanique. Celle de Daniel Knight revient sur le rapport des Grecs à leur (non-)appartenance aux Balkans. L'auteur montre que cette appartenance se construit en fonction de la façon dont les Grecs perçoivent leur situation économique, nous permettant d'entrevoir une autre définition des Balkans, formée par l'expérience de la pauvreté, des difficultés économiques et de la débrouille. En questionnant l'identité de genre d'un groupe de féministes bosniennes, le chapitre d'Elissa Helms interroge ce que peut signifier être une femme musulmane ou balkanique. Enfin, le chapitre de Nataša Gregorič Bon questionne le rapport des Albanais à leurs origines et la façon dont les discours sur les Balkans et l'Europe mettent en jeu les relations aux héritages ottoman et communiste.

4 Un autre intérêt de l'ouvrage réside dans l'attention portée aux transitions entre socialisme et postsocialisme dans la région. Ainsi le chapitre de Carolin LeutloffGrandits étudie les réseaux de soutien et d'entraide familiale, notamment les formes de sociabilité, de garde d'enfants et les soins procurés aux parents, entre les années 1980 et 2010 dans trois communautés rurales et périurbaines en Croatie et au Kosovo, des sociétés connaissant d'importants phénomènes migratoires. Le chapitre d'Azra Hromadžić apporte un éclairage utile sur cette même thématique générale à partir d'une étude sur une maison de retraite à Bihać, en Bosnie-Herzégovine. L'auteur met en lumière la privatisation des réseaux d'entraide et l'évolution des possibilités de subvenir aux besoins familiaux entre socialisme et postsocialisme. Le chapitre de David Montgomery sur la relation des Albanais à la religion revient sur les transformations $\mathrm{du}$ fait religieux en Albanie dans l'après-socialisme, entre répression de la religion sous Hoxha et retour du religieux par des circuits étrangers à partir de 1990. Cette contribution interroge également le rapport individuel au religieux et la façon dont celui-ci participe à redéfinir les rôles sociaux. Le chapitre d'Alyssa Grossman porte sur l'émergence de lieux de mémoire spontanés à Bucarest et sur les différentes strates urbanistiques qui composent la ville. Les traces architecturales du passé demeurent, comme par exemple des fenêtres anciennes, des publicités ou des vitrines de magasin, rappelant des scènes du quotidien et qui appartiennent dorénavant au passé. Enfin, Andrew Konitzer se penche sur le parcours d'un homme vivant dans un village serbe, passant d'employé d'une entreprise à l'époque socialiste à entrepreneur dans l'aprèssocialisme. Konitzer étudie ainsi l'impact des transformations économiques et politiques qu'a connues la Serbie dans les années 1990 en se basant sur le parcours de 
cet homme dont les savoir-faire et l'expérience lui permettent de s'en sortir dans un environnement économique dégradé.

5 L'ouvrage questionne également l'émergence des identités collectives et nationales. En s'intéressant notamment aux liens entre café, orientalisme et Balkans en Bulgarie, Mary Neuburger nous explique dans son chapitre comment les Bulgares se définissent en tant que nation balkanique par la culture du café turc. Cette culture fortement orientalisée différencierait la Bulgarie du reste de l'Europe. Ainsi, la période ottomane, définie par ailleurs comme un asservissement et une occupation, serait aussi romantisée et vue comme une matrice dans laquelle s'est forgée l'identité bulgare. Le chapitre de Keith Brown porte quant à lui un regard sur la construction des identités macédonienne et slovène par l'attachement ou le rejet d'un plat: le burek. En Macédoine, le burek est un symbole identitaire fort de la culture macédonienne. En Slovénie, le burek est stigmatisé dans les discours comme un plat oriental et évoqué pour distancier la Slovénie de ses voisins du sud, alors même que ce feuilleté est omniprésent dans le quotidien. Le questionnement sur l'identité est également visible dans le chapitre de Roman Kuhar, travaillant sur la relation des personnes homosexuelles avec leur famille en Slovénie. Cette contribution permet de voir comment un discours d'acceptation de l'homosexualité est mis en avant pour montrer le rattachement de la Slovénie à l'Europe occidentale. Le chapitre de Jelena Džankić s'intéresse quant à lui à l'émergence d'une identité monténégrine distincte de l'identité serbe. Il revient également sur la crise économique et les événements politiques des années 1990 et 2000 ayant mené à l'indépendance du pays en 2006. Dans son chapitre, Monika Palmberger aborde la construction identitaire d'un point de vue territorial. Elle analyse la façon dont les jeunes de Mostar se situent vis-à-vis des transformations qu'a connues la ville depuis les années 1990, et notamment sa division en deux secteurs. Les différentes générations n'évaluent pas l'impact de la guerre de la même façon. L'auteure interprète la distanciation personnelle envers le politique et la guerre comme une volonté de retirer son histoire personnelle d'une histoire globale. La contribution d'Ilká Thiessen montre que l'identité macédonienne se construit à travers des formes urbaines émergentes, en particulier la mise en place du projet Skopje 2014.

6 L'ouvrage se penche aussi sur les relations entre arts de vivre et identités au quotidien. Le chapitre de Frances Trix se concentre sur la pratique du jeûne du ramadan à Prizren, au Kosovo. L'auteure démontre qu'il est possible d'étudier la ville à travers la pratique du ramadan par ses habitants, et ceci de la période socialiste à la période contemporaine. Le chapitre de Carol Silverman met l'accent sur la place de la musique de mariage en Bulgarie. Silverman retrace le parcours de ce genre musical, entre rejet de la part des pouvoirs publics sous le communisme, recul dans les années 1990 face au développement d'une nouvelle pop-folk, la chalga, et retour en force depuis les années 2000 à travers son assimilation par la chalga et la marchandisation du secteur. Dans sa contribution, Čarna Brković revient sur la démarcation de la frontière sur la rivière Drina entre la Bosnie et la Serbie pour interroger la façon dont est perçue cette frontière mouvante au quotidien et comment celle-ci se construit à travers l'expérience personnelle des habitants.

7 Pour conclure, Everyday Life in the Balkans sera un ouvrage utile pour ceux qui souhaitent acquérir des connaissances sur les sociétés et l'histoire de la région. L'ouvrage se révèle plutôt être un recueil rassemblant de nombreuses recherches sur la vie quotidienne dans les Balkans qu'une exploration approfondie de la notion de vie 
quotidienne dans les Balkans. Si la première partie est clairement historique, les contributions suivantes incitent à questionner tant la linéarité et les continuités que les ruptures chronologiques qu'a connues la région. Souvent, au sein d'un même ensemble thématique, les liens entre les chapitres sont ténus, ce qui nourrit une impression de dispersion et de manque d'unité. Il n'en reste pas moins que grâce au grand nombre de contributions, à la variété des terrains, des sujets et des approches disciplinaires, cet ouvrage atteint son objectif de brosser un portrait complexe et dynamique des sociétés balkaniques.

\section{NOTES}

1. MONTGOMERY David (ed.), Practicing Islam: Knowledge, Experience, and Social Navigation in Kyrgyzstan, Pittsburgh, University of Pittsburgh Press, 2016.

2. MONTGOMery David (ed.), Everyday Life in Central Asia: Past and Present, Pittsburgh, University of Pittsburgh Press, 2007.

3. GOLDSTEIN Piotr, "Grassroots Narratives and Practices of Diversity in Mostar and Novi Sad", dans Tatjana Matejskova, Marco Antonsich (dir.), Governing through Diversity: Migration Societies in Post-Multiculturalist Times, Londres, Palgrave Macmillan, 2015, p. 104-124.

4. LUTHAR Breda, PUŠNIK Maruša (eds), Remembering Utopia: Culture of Everyday Life in Yugoslavia, Washington DC, New Academia Publishing, 2010.

5. STRAUSS Johann, «Linguistic Diversity and Everyday Life in the Ottoman Cities of the Eastern Mediterranean and the Balkans (Late 19th-Early 20th Century) », The History of the Family, vol. 16, $\mathrm{n}^{\circ}$ 2, 2011, p. 126-141.

6. RORY Archer, « Assessing Turbofolk Controversies: Popular Music between the Nation and the Balkans ", Southeastern Europe. L'Europe du Sud-Est, vol. 36, nº 2, 2012, p. 178-216.

INDEX

Index géographique : Balkans

\section{AUTEURS}

MEHDI BELASRI

Cetobac, EHESS, Paris 